\title{
Enteral nutritional support via the mucous fistula: feasibility and safety in post-operative patients
}

\author{
C. M. Lever ${ }^{1}$, L. Vokes ${ }^{2}$, J. Pratt ${ }^{2}$ and T. M. Trebble ${ }^{1}$ \\ ${ }^{1}$ Department of Gastroenterology, Queen Alexandra Hospital, Portsmouth, PO6 3LY, UK and ${ }^{2}$ Department of Nutrition, \\ Queen Alexandra Hospital, Portsmouth PO6 $3 L Y, U K$
}

\begin{abstract}
Abdominal surgery with small bowel (SB) resection may require delayed reanastomosis with the formation of a temporary defunctioning end stoma and mucous fistula (MF). If cited proximally, this may result in a short bowel in the proximal limb with redundant longer bowel in the distal limb. Oral/nasogastric intake may induce uncontrolled stomal fluid ( $>2.5$ litres/d) and electrolyte losses leading to prolonged hospital admission or parenteral support. The distal bowel can be utilised for absorption of nutrition, fluids and electrolytes if sufficient in length and accessible via the MF. However, the viability and safety of small bowel MF enteral support have been poorly reported. We present a case series of our experience in a busy district general hospital.

Referred post-operative patients with an MF who demonstrated metabolic and nutritional instability on oral/NG intake due to uncontrolled stoma losses were assessed for MF feeding. A 14 French Foley catheter was inserted into the MF through a universal catheter access port placed into a windowed stoma bag and connected to standard enteral feeding apparatus. Elemental feed (EO28), rehydration fluids, electrolytes or nutritional supplements were administered to meet the patient's requirements using standard methods of jejunal feeding. Proximal stoma output was monitored and oral dietary intake reduced if necessary.

Between February 2007 and October 2008, eight patients were found to be appropriate for a trial of MF feeding. All patients had undergone SB resection with residual proximal small bowel length ranging from $75 \mathrm{~cm}$ to $150 \mathrm{~cm}$ (mean $116 \mathrm{~cm}$ ) from the DJ flexure; all had distal SB length greater than $1 \mathrm{~m}$ with a viable large bowel in continuity. Patients reported initial difficulties in assembling the feeding tube and access port via the stoma window, however with training all gained competency allowing home discharge with telephone and clinic support. The universal catheter access port allowed a water tight seal, and leakage around the feeding tube was negligible. There were no major metabolic complications of MF feeding including those patients who remained on controlled oral intake. All patients received enteral support for a minimum of 3 months prior to assessment for surgical re-anastomosis.

MF feeding may be a safe and effective method for nutritional support in appropriate patients and may allow home discharge in the presence of a high output proximal stoma. The use of a Foley catheter as a feeding tube and a simple catheter port allowed inexpensive and simple means of access. However, dedicated training, support and monitoring may be required to allow home discharge.
\end{abstract}

